\title{
Erratum
}

\section{Emergence of immune escape variant of mammary tumors that has distinct proteomic profile and a reduced ability to induce "danger signals"}

Masoud H. Manjili, Hilal Arnouk, Keith L. Knutson, Maciej Kmieciak, Mary L. Disis, John R. Subjeck, and A. Latif Kazim

Breast Cancer Research and Treatment DOI 10.1007/s10549-005-9044-4

In Table 1 of the original, the column headings were missing and the first row was erroneously set in their position. The missing column headings are as follows: Column 1: spots; Column 2: protein ID; Column 3: accession number; Column 4: score; Column 5: peptides matched; Column 6: molecular weight (kDa); Column 7: IP; Column 8: coverage \%; Column 9: fold difference; Column 10: function. 